\title{
Representation, space and Hollywood Squares: looking at things that aren't there anymore
}

\author{
Daniel C. Richardson*, Michael J. Spivey \\ Department of Psychology, Cornell University, Ithaca, NY 14853, USA
}

Received 9 September 1999; received in revised form 26 January 2000; accepted 12 April 2000

\begin{abstract}
It has been argued that the human cognitive system is capable of using spatial indexes or oculomotor coordinates to relieve working memory load (Ballard, D. H., Hayhoe, M. M., Pook, P. K., \& Rao, R. P. N. (1997). Behavioral and Brain Sciences, 20(4), 723), track multiple moving items through occlusion (Scholl, D. J., \& Pylyshyn, Z. W. (1999). Cognitive Psychology, 38, 259) or link incompatible cognitive and sensorimotor codes (Bridgeman, B., \& Huemer, V. (1998). Consciousness and Cognition, 7, 454). Here we examine the use of such spatial information in memory for semantic information. Previous research has often focused on the role of task demands and the level of automaticity in the encoding of spatial location in memory tasks. We present five experiments where location is irrelevant to the task, and participants' encoding of spatial information is measured implicitly by their looking behavior during recall. In a paradigm developed from Spivey and Geng (Spivey, M. J., \& Geng, J. (2000). submitted for publication), participants were presented with pieces of auditory, semantic information as part of an event occurring in one of four regions of a computer screen. In front of a blank grid, they were asked a question relating to one of those facts. Under certain conditions it was found that during the question period participants made significantly more saccades to the empty region of space where the semantic information had been previously presented. Our findings are discussed in relation to previous research on memory and spatial location, the dorsal and ventral streams of the visual system, and the notion of a cognitive-perceptual system using spatial indexes to exploit the stability of the external world. (C) 2000 Elsevier Science B.V. All rights reserved.
\end{abstract}

Keywords: Memory; Eye movements; Visual attention; Spatial representation; Embodiment

\footnotetext{
* Corresponding author. Tel.: +1-607-255-6398; fax: +1-607-255-8433.

E-mail address: dcr18@cornell.edu (D.C. Richardson).
} 


\section{Introduction}

Many of us have had the experience of trying to remember a piece of information from a textbook when we cannot recall the right chapter, or even the particular context it appeared in, but we do have a notion that it appeared in a particular region on the page. We then flick through the book scanning only, say, the top portion of the left hand page. One of our colleagues recalls that when he was stuck on a question in a high school math exam, he would stare at the blank region of the blackboard where the teacher had written - perhaps weeks earlier - the formula he needed to recall.

These experiences suggest that there is a special relationship between spatial information and memory. In the theory of deictic coding proposed by Ballard, Hayhoe, Pook and Rao (1997), it is argued that 'pointing behaviors', such as eye fixations, provide an efficient way for the cognitive system to tag mental representations. Similarly, Pylyshyn and colleagues (e.g. Pylyshyn, 1989, 1994; Pylyshyn \& Storm, 1988; Scholl \& Pylyshyn, 1999) claim that the simple identity, or objecthood, of multiple items can be tracked by the use of FINST ('finger instantiation') indexes. Perhaps a mechanism such as cell assemblies (Hebb, 1968; Pulvermüller, 1999) exploits the temporal association between a perceptual input and the corresponding oculomotor coordinates to establish a spatial index for the cross-modal representation. Our current theoretical motivation was to find evidence that spatial indexes are being employed by the cognitive system, even in a memory task where location is irrelevant.

In the five experiments reported here, we presented participants with auditory, semantic information in various visual locations. Studies such as Ballard, Hayhoe and Pelz (1995) suggest that the eye tracking methodology may be a useful way to investigate the role of memory and spatial indexes in natural tasks. Participants' encoding of spatial information was revealed by their looking behavior when answering a question that related to information that had previously been presented in a (now empty) region of space.

It is increasingly acknowledged that eye movements are an important source of evidence in the study of cognition. As Bridgeman (1992, p. 76) remarks, "the vast majority of behavioral acts are saccadic jumps of the eye, unaccompanied by any other behaviors". Current research further demonstrates that eye movements can provide a valuable window on the time course of cognitive processing and the activation of partial representations (e.g. Tanenhaus, Spivey-Knowlton, Eberhard $\&$ Sedivy, 1995). In these situations, it appears that eye movements are rarely under voluntary control. For example, in one of their experiments, Tanenhaus et al. (1995) asked participants to 'pick up the candy' amongst an array of other objects. Unaware that they had done so, participants would often saccade to an object with similar phonology, such as a candle (see also Allopenna, Magnuson \& Tanenhaus, 1998; Spivey \& Marian, 1999).

Our current concern, the encoding of spatial location in memory, has been the subject of much research. Often issues of automaticity, task demands and strategies are raised. Perhaps then using eye tracking methodologies in this domain would prove fruitful, since they provide an implicit measure of representations and cogni- 
tive processes. It may well be that the introductory anecdote of strategically scanning an empty blackboard is importantly different from the implicit aspects of memory that we are studying with the eye tracking methodology in the current experiments. Recent research is demonstrating interesting dissociations between memory effects that participants explicitly report and the implicit memory that their eye movements reveal. For example, Neal J. Cohen and colleagues (Althoff \& Cohen, 1999; Cohen, Ryan \& Althoff, 1999) repeatedly presented a number of pictures to participants, occasionally making a change to the image in the third presentation. Whilst participants were typically 'change blind' to these alterations and unable to report any difference, their eye movements would center on the location of the change. This suggests that there is an aspect of memory which is below the level of explicit awareness, and hence, we presume, intentional strategies, yet accessible with eye tracking methodologies. The motivation for the current studies then is to examine how this aspect of implicit memory may contribute to spatial encoding of semantic information.

Recently, Spivey and Geng (2000) reported finding systematic saccades to particular blank regions of space in a memory task. In their second experiment, participants saw four shapes of varying color and orientation in four corners of a $3 \times 3$ grid. The screen went blank for a moment, and then only three of the shapes returned (see Fig. 1). Participants were asked a question about the orientation or color of the missing shape. They found that in 30-50\% of the trials, participants made a saccade to the blank location of the grid where the queried shape had once been, despite the fact that there was clearly no useful information present there. They suggested that the question may trigger oculomotor parameters that accompanied the forming of the visual memory for that shape.

In the experiments reported here, we attempted to extend this finding with object properties to spatial location and semantic memory of linguistic information. In the Hollywood Squares experiments, we presented participants with four video clips or
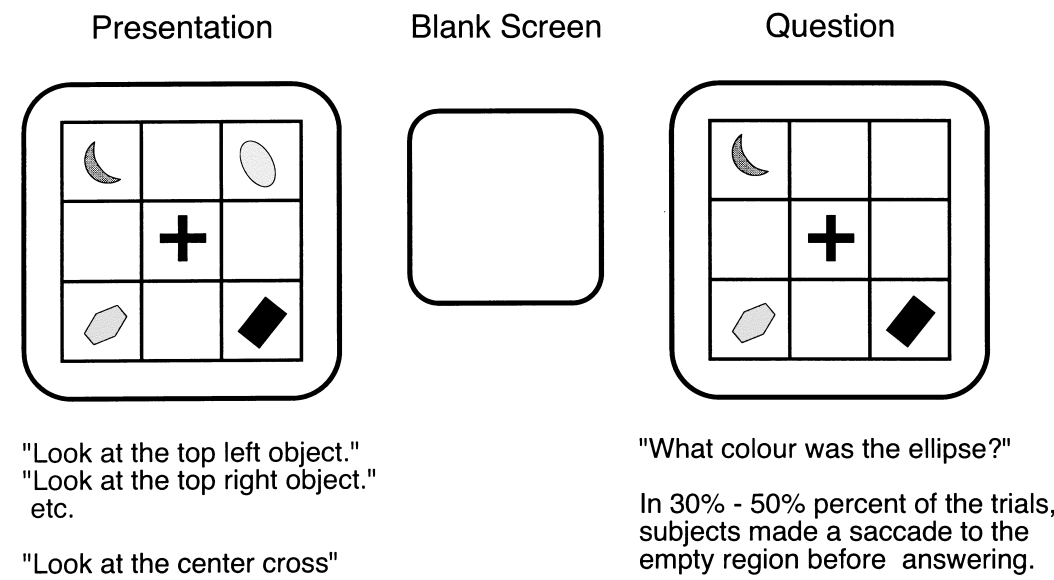

Fig. 1. Spivey and Geng (2000) - schematic of Experiment 2. 
animations that contained spoken information. ${ }^{1}$ The events occurred one at a time in random order in the four locations of a $2 \times 2$ grid and disappeared. A question then probed the participant's memory of the semantic information conveyed in one of the events. We hypothesized that even in front of a completely blank grid, participants would make systematic saccades to the region of space where they perceived the event.

It is known that primates can launch eye movements to the location of remembered targets (Gnadt, Bracewell \& Andersen, 1991), that spatial location can be used as a cue for memory recall (Sinclair, Healy \& Bourne, 1997; Winograd \& Church, 1988), and that readers can make accurate, long distance regressive eye movements to queried words (Kennedy \& Murray, 1987); therefore, it might not seem too unreasonable to hypothesize that saccades could be launched to semantic associates of remembered targets. The work of Fendrich (1998) and others suggests that the spatial component of a motoric response can serve as a recognition cue. So, if we can characterize eye movements as a motor pattern like any other, then this would indicate that oculomotor coordinates could become associated with a memory. Indeed, Bradley, Cuthbert and Lang (1988) have demonstrated that eye movement patterns (vertical or horizontal) can act as a contextual cue in recognizing digit strings. In general, one might expect eye position during encoding to be reinstated during recall because of the many similar effects in context-dependent memory research (e.g. Bjork \& Richardson, 1989; Godden \& Baddeley, 1975; Winograd \& Church, 1988). Typically, the criterion for context-dependent memory effects in the literature is an increase in accuracy during recall when the encoding context is reproduced. Therefore, this literature would predict memory to be improved when eye position is reinstated compared to when it is not.

However, there are a number of reasons why one might not expect an observer to look at a blank region that was associated with the presentation of some semantic propositional knowledge. It is still the case that the visual input during a fixation of an empty region of space does not provide any directly useful information at all. Moreover, Spivey and Geng (2000) report evidence that saccades to blank regions of space are more closely associated with (and therefore more readily triggered by) representations of spatial relations, such as tilt, than with less spatially relevant representations of intrinsic object properties, such as color. This would not bode well for the Hollywood Squares hypothesis. Furthermore, much of the work on memory and spatial location stresses that entirely different processes may be involved in location memory for objects than for written words (cf. Pezdek, Roman \& Sobolik, 1986). In the Hollywood Squares experiments, we are not even using semantic information that appears visually, but spoken facts that are associated with a visual event. Lastly, Glenberg, Schroeder and Robertson (1998) published a paper entitled 'Averting the gaze disengages the environment and facilitates remembering' which, on the surface at least, would suggest that during seman-

\footnotetext{
${ }^{1}$ In the gameshow 'Hollywood Squares', celebrities sit in a grid like formation and offer answers to questions that the contestant guesses are correct or incorrect. Based on this vague resemblance, the current studies got their name.
} 
tic knowledge recall, eye movements are likely to avoid risking any interference from visual surroundings.

In the general discussion, we connect our findings with neurophysiological and behavioral research on the primate visual system. We will argue that it is possible to interpret our results in terms of the visual system relying on the stability of the external world, as discussed by researchers such as Bridgeman and O'Regan (e.g. Bridgeman, Van der Heijden \& Velichkovsky, 1994; O'Regan, 1992).

\section{Experiment 1}

For ease of exposition, throughout this paper we will be referring to the location of the event which is being probed in memory as the 'critical location' or 'critical port'. In each study presented here, four facts are associated with four locations, and a question is asked about one of those facts. The location which contained information relevant to the question is called the 'critical port'. In all the following studies then, we are interested in whether or not the participants look significantly more often to the critical port than the other ports. Importantly, at the moment in time we are looking for saccades, all the ports are undifferentiated blank regions in a $2 \times 2$ grid.

\subsection{Method}

\subsubsection{Participants}

Eighteen Cornell undergraduate students took part in the study in exchange for course credit. All had normal vision, or vision corrected by soft contact lenses.

\subsubsection{Apparatus}

Eye movements were monitored by an ISCAN eye tracker mounted on top of a lightweight headband. The eye camera provided an infrared image of the left eye sampled at $60 \mathrm{~Hz}$. The center of the pupil and the corneal reflection were tracked to determine the direction of the eye relative to the head. A scene camera, yoked with the view of the tracked eye, provided an image of the participant's field of view. Gaze position (indicated by crosshairs) was superimposed over the scene camera image and recorded onto a Hi8 VCR with $30 \mathrm{~Hz}$ frame-by-frame playback. The accuracy of the gaze position record was approximately $0.5^{\circ}$ of visual angle. The video record was synchronized with the audio record for all data analysis.

The calibration grid and stimuli were presented on an Apple Macintosh 7200/90 computer running Psyscope 1.22 (Cohen, MacWhinney, Flatt \& Provost, 1993) with a 20 inch monitor and external speakers.

\subsubsection{Stimuli}

Eight sets of four short factual statements were constructed. The facts were either general knowledge from an encyclopedia (e.g. 'Although the largest city in Australia is Sydney, the capital is Canberra') or were short statements about fictional characters (e.g. 'Claire gave up her tennis career when she injured her shoulder'). The 
questions were statements corresponding to each fact that could be true or false (e.g. 'Sydney is the capital of Australia').

Each set of four facts was associated with four talking heads. We used high school students from across the country who were attending Cornell's Summer School program. This ensured that our participants did not recognize the people in our stimuli. The talking heads were recorded speaking each fact in the set looking into a video camera. These recordings were converted to MPEG files which could be displayed on the Apple Macintosh computer. The questions were recorded as sound files by the experimenter. This gave us 128 MPEG video clips and 32 audio clips. From this set, each subject was presented a total of 32 MPEGs and eight audio clips - four facts and one question per trial.

During the experiment, the computer screen was divided into a $2 \times 2$ grid, subtending $32.3 \times 25.8^{\circ}$ of visual angle. The four regions of the grid are referred to as 'ports', each subtending $16.3 \times 12.9^{\circ}$ of visual angle. Each MPEG appeared in one of the four ports, subtending $8.3 \times 5.9^{\circ}$ of visual angle. Fig. 2 presents a schematic of the stimuli and the experimental procedure.

\subsubsection{Procedure}

To mask our hypothesis, the participant signed a consent form that suggested our study was concerned with how effectively different people convey information. The eye tracker was then calibrated using a $3 \times 3$ grid of red dots on the computer monitor, a procedure that typically took between 5 and $10 \mathrm{~min}$.

There were eight trials consisting of a random order of the eight sets of facts. In a trial, the participant was presented with four MPEG video clips. Each one appeared in one of the four ports, played for an average of $6 \mathrm{~s}$, and then disappeared. The speaker, order of facts and locations were all randomized, such that the participant saw four facts appear in four different ports, read by four different speakers. The participant only saw each speaker and heard each fact once.

After the fourth fact, the participant heard a pre-recorded statement while looking at a blank grid. The statement referred to one of the four facts, chosen at random. The participant judged the statement to be true or false in relation to the fact, and said her reply out loud (the response was recorded by a microphone). She then pressed the space bar to initiate the next trial. ${ }^{2}$

\subsection{Results}

The participants' eye movements were analyzed in the period beginning from the onset of the question and ending with the participant's response. The number of fixations in each port was counted. A fixation was operationalized as steady eye position crosshairs in one of the four ports for $100 \mathrm{~ms}$ or more (three frames on the video tape). The criterion of $100 \mathrm{~ms}$ was chosen to exclude crosshair movement that

\footnotetext{
${ }^{2}$ Online at http://susan.psych.cornell.edu/hollywood.html one can view example trials from each experiment, as seen through the eye tracker's scene camera with the participant's fixation crosshairs imposed.
} 


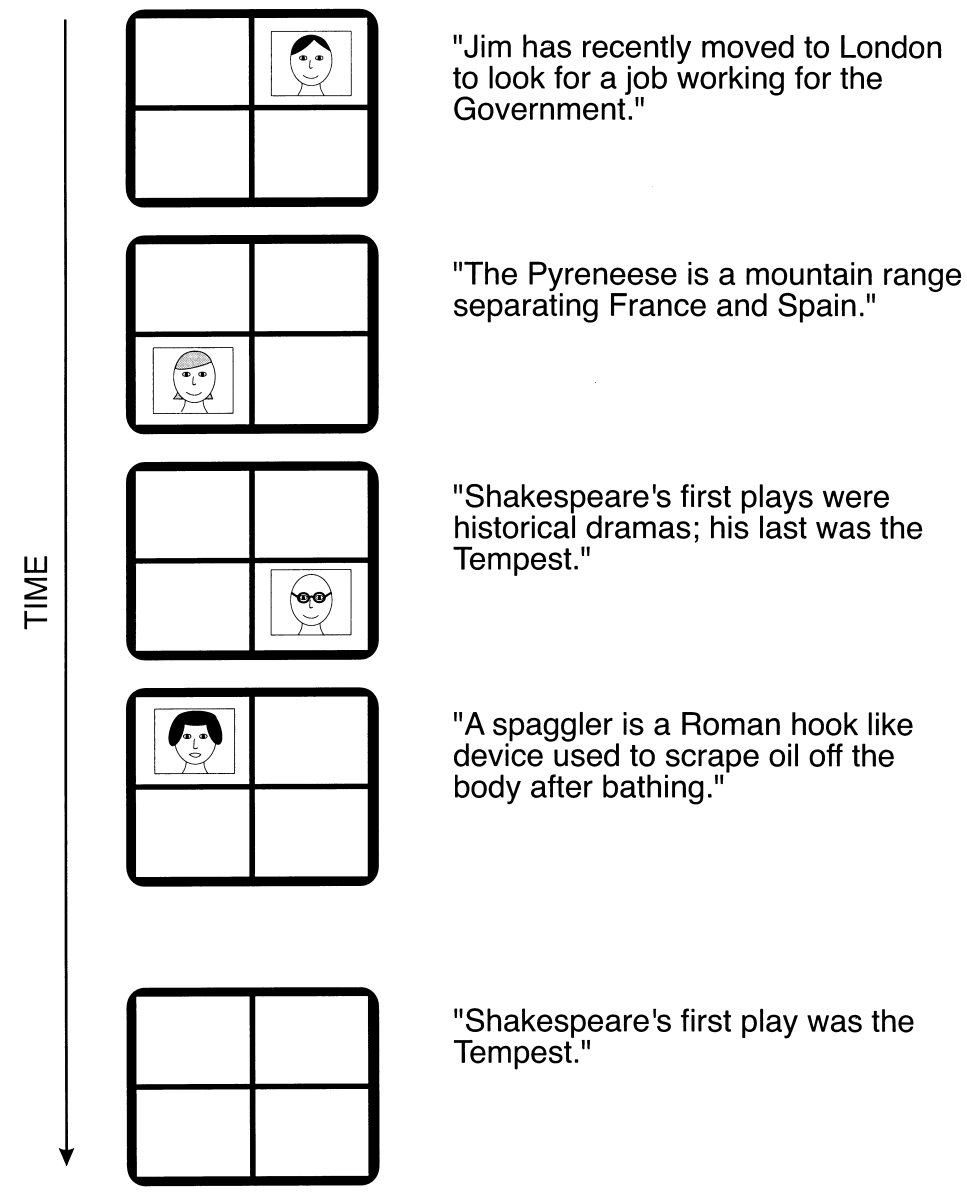

Fig. 2. Schematic of a trial in Experiment 1. The participant is presented with four facts read by different speakers, appearing randomly in each of the ports. Then, while looking at a blank grid, the participant hears a true or false statement relating to one of the four facts, and replies true or false. In this example, the critical port would be bottom right.

was due to blinking or saccades across a port. On average, there were about 2.04 fixations per trial. The participants' true/false responses were also coded.

The data were then 'clock coded'. In each trial, the port where the relevant fact appeared, the 'critical port', was labeled 0 . The other three ports were labeled 1-3, going around in a clockwise direction. Our hypothesis then is that there will be more looks to port 0 than the other ports.

Note that since the port labeling changes according to the location of the critical port, which is randomly positioned for each trial, any absolute looking bias the participants might have - casting their gaze slightly downward during their answers, for example - should be distributed across all the clock coded ports. 
We analyzed the mean fixations per trial using a repeated measures ANOVA. There was a significant effect of port $(F(3,51)=2.97, P<0.05)$. Participants made more looks to the critical port (the mean number of fixations per trial was 0.73 ) than the other ports $(0.44,0.40$ and 0.47 for ports 1,2 and 3 , respectively). The mean fixations per trial are summarized in Fig. 3. Tukey tests of pairwise contrasts between the critical port and ports 1, 2 and 3 reached significance (all $P<0.05$ ). Moreover, the pattern of more looks to the critical port seemed to be consistent across participants: 13 out of 18 showed the effect. Participants' accuracy in answering the questions was analyzed by whether they made zero looks to the critical port, or one or more looks. Although participants were slightly more accurate when they had made one or more looks to the critical port (83\% correct versus $73 \%)$, this effect did not approach significance $(F(1,17)=1.59, P>0.2)$. In debriefing, participants either professed no knowledge of our hypothesis, or were confident that their looking behavior was relevant only during the fact presentation stage.

\subsection{Discussion}

These results show that the participants' looking behavior was not random: there was a significant tendency to look at the blank region of space where the probed information had been originally presented. Yet the accuracy data suggest that memory for that semantic information did not improve when participants made a saccade to the correct location - which would have been expected if the looking behaviors were solely the result of a contextual memory effect - and adds to

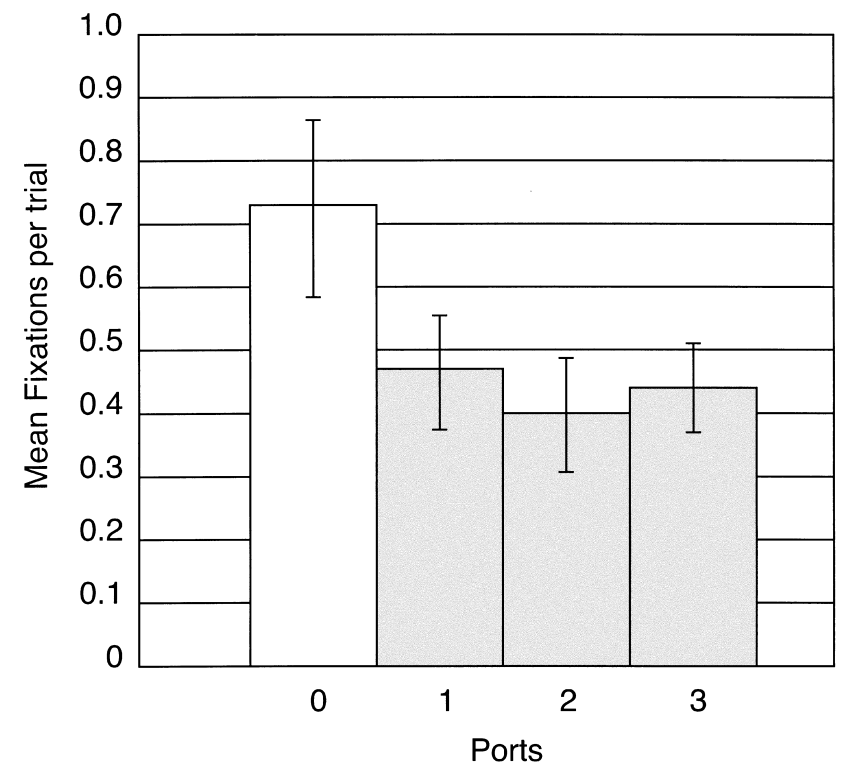

Fig. 3. Mean fixations per trial, Experiment 1 (error bars represent pooled standard errors). 
previous findings that item memory is independent of location information (e.g. Pollatsek, Rayner \& Henderson, 1990).

With Experiment 1, the finding of Experiment 2 in Spivey and Geng (2000) has successfully been extended to auditory, semantic information that is associated with a spatial location. Participants were not explicitly questioned about any visual properties of the events, and were not even aware that their spatial knowledge was being tapped during the question period. This preliminary finding suggests that spatial indexes may be being attached to semantic representations with a substantial degree of automaticity.

However, it could be argued that the effect we have found is not due to a direct connection between spatial and semantic representations. Perhaps participants are using some sort of mnemonic strategy of associating the factual information with something about the physical appearance of the speaker. They might imagine the speaker playing tennis, if the fact concerns 'Claire's tennis career', for example. Thus, spatial information is in fact explicitly brought into play by being part of the representation of that particular speaker. This interpretation might follow from the conclusion by Pezdek et al. (1986) that spatial information is more easily tied to visual objects than to words. In Experiment 2 then, we removed any visual differences between the fact presentations, such that any looking behaviors would be generated purely by a relationship between spatial and auditory semantic information.

\section{Experiment 2}

This study closely followed the paradigm of Experiment 1. However, instead of visually rich MPEGs of talking heads, participants saw a spinning cross appear in the various ports while listening to sound clips of the facts. In this way, spatial location would be the only feature that visually distinguishes the four factual presentations.

\subsection{Method}

\subsubsection{Participants}

Sixteen Cornell undergraduates took part in the study in exchange for course credit. All had normal vision, or vision corrected by soft contact lenses.

\subsubsection{Stimuli}

Twelve sets of four facts and questions were generated using the same procedure as Experiment 1. The facts were read out by one person and recorded as sound files.

\subsubsection{Procedure}

The design was the same as Experiment 1, except that there were 12 trials, and the MPEGs of speakers were replaced by a spinning cross for the duration of the fact sound file. The cross was approximately the same size as the MPEGs and spun slowly while cycling between red and black. The participants were told to fixate on the spinning cross whenever it appeared, and that the eye tracker was being used 
to check their performance. Analysis of the video tape showed that participants complied with these instructions.

\subsection{Results}

The data were coded in the same way as Experiment 1, and the mean fixations per trial were subjected to a repeated measures ANOVA. There were, on average, 2.83 fixations per trial. As before, there was a significant effect of port $(F(3,45)=5.585$, $P<0.005$ ). Participants made more looks to the critical port (the mean number of fixations was 0.96 per trial) than the other ports $(0.63,0.54$ and 0.70 for ports 1,2 and 3, respectively). The mean fixations per trial are summarized in Fig. 4. Tukey tests of pairwise contrasts between the critical port and ports 1, 2 and 3 reached significance (all $P<0.05$ ). A large majority of participants, 14 out of 16 , showed this pattern of behavior. Participants' accuracy in answering the questions was slightly lower (73\% correct) when they had made one or more looks to the critical port compared to having made no looks at all ( $83 \%$ correct); however, this effect did not approach significance $(F(1,15)=0.141, P>0.7)$. In debriefing, participants confirmed that they had believed our cover story that the eye tracker was only present to check their compliance with instructions during the fact presentation phase.

\subsection{Discussion}

In this second experiment, each fact was presented with a visually identical spinning cross, and yet we still found a significantly higher number of looks to the critical port compared to the other ports. This result demonstrates that looks

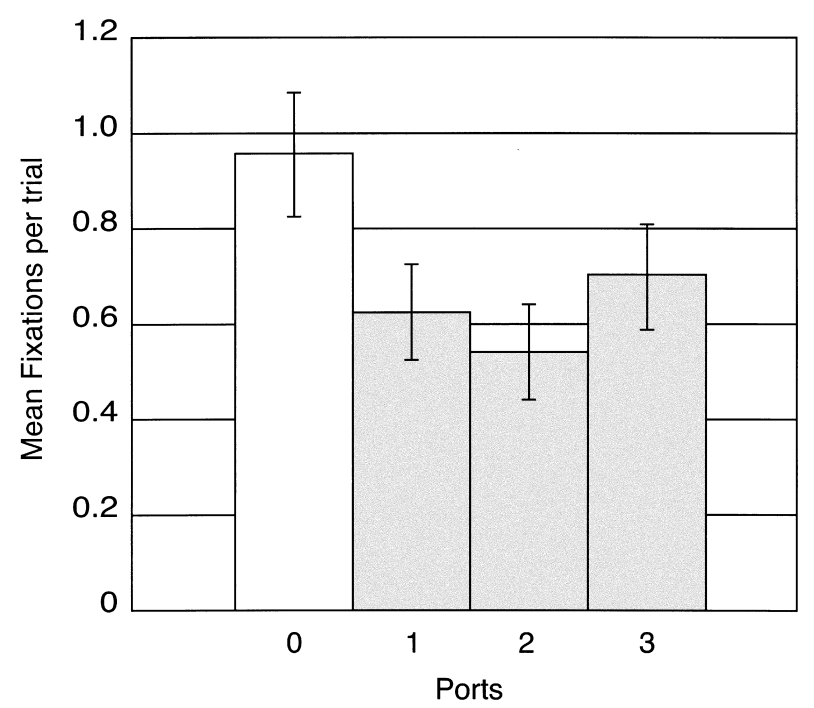

Fig. 4. Mean fixations per trial, Experiment 2 (error bars represent pooled standard errors). 
to a blank region are not necessarily related to any visual feature of the event that occurred there. Thus, the effect cannot be explained by participants using any sort of mnemonic strategy linking the physical appearance of the talking head with the fact. Rather it seems that auditory, semantic information is being directly associated, or tagged, with spatial information. Accordingly, we decided to investigate the nature of this spatial representation.

Spivey and Geng (2000) suggested an explanation of their finding in terms of a stored visual representation triggering the "oculomotor parameters that accompanied the forming of that visual memory". We might usefully ask then whether the oculomotor parameters themselves are stored as part of the visual memory, or whether a more abstract (non-motor) spatial representation is triggered, which is then translated during the probe question into eye movements to the critical port. Experiment 3 was a first step in addressing this question.

\section{Experiment 3}

Here we were interested in the contribution of specifically oculomotor information during the formation of memories of the facts. Experiment 3 used the same stimuli and design as Experiment 1, but the participant's eye movements were controlled during presentation of the facts by means of a central fixation cross.

\subsection{Method}

\subsubsection{Participants}

Twenty-eight Cornell undergraduate students took part in the study in exchange for course credit. All had normal vision, or vision corrected by soft contact lenses.

\subsubsection{Stimuli and procedure}

The experiment was exactly the same as Experiment 1 with one difference: during the presentation of each MPEG, a cross appeared in the center of the screen (see Fig. 5). The cross spun slowly and cycled in color from red to black. The participants were told to fixate on the spinning cross whenever it appeared, and that the eye tracker was checking their compliance. In between the presentation of each fact, and during the question period, the cross was not present.

\subsection{Results}

The data were analyzed in the same way as Experiment 1. On average, 1.3 fixations were made on each trial. A repeated measures ANOVA of mean fixations per trial revealed that the effect of port did not approach significance $(F(3,81)=1.239, P>0.3)$. Indeed, participants made slightly fewer looks to the critical port on average (the mean number of fixations per trial was 0.26 ) than the other ports $(0.35,0.38$ and 0.28 for ports 1,2 and 3 , respectively). The mean fixations per trial are summarized in Fig. 6.

For the analysis of the accuracy of participants' answers, ten participants had to be 
Fact Presentation

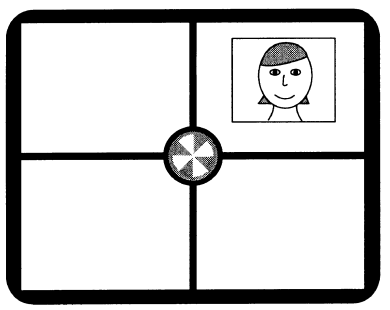

Subject fixates the animated center cross

\section{Question Period}

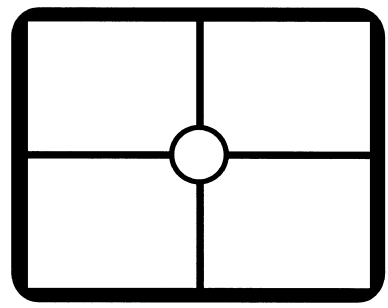

No cross is present

Fig. 5. Diagram of the grid and fixation cross used in Experiment 3.

excluded since they never made a look to the critical port. Those that did were somewhat less accurate when they had made a look to the critical port (63\%), compared to when they had made no looks to the critical port (78\%). However, this effect did not approach significance $(F(1,17)=2.728, P>0.1)$. This suggestive difference may be due to participants looking all over the grid when they happen to have trouble answering the question.

\subsection{Discussion}

The results of Experiment 3 suggest that fixations on each port during presentation

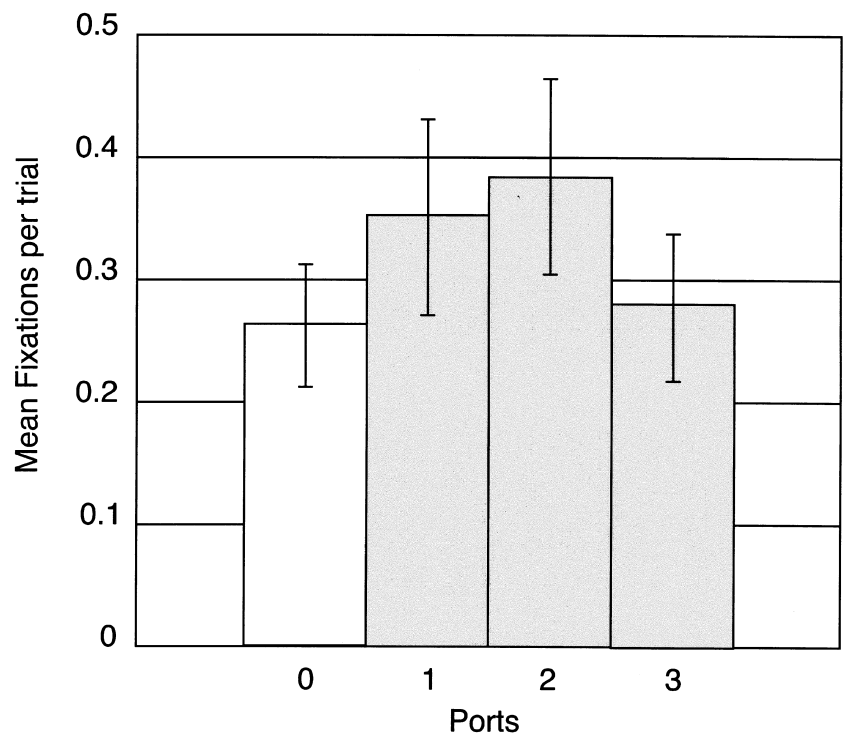

Fig. 6. Mean fixations per trial, Experiment 3 (error bars represent pooled standard errors). 
of the facts may be necessary for the effect found in Experiments 1 and 2. However, we cannot definitively conclude that it is stored oculomotor coordinates that generate a look to the critical port during the question period. It might be that participants were completely ignoring the MPEG clips while they fixated on the central cross. That is to say, if covert visual attention were not being allocated to the MPEG clips, then not even an abstract (non-motor) spatial representation would be available to trigger an eye movement. If this were the case, then it would not be at all surprising that we found no evidence of a memory for location in the eye movement patterns (cf. Logan, 1994).

\section{Experiment 4}

The results of Experiment 3 might be obtained if participants ignored all visual stimuli except the fixation cross, and simply listened to the auditory stimuli. In Experiment 4 then, we attempted to ensure that participants were attending to the MPEG presentation parafoveally. The participants were given the additional task of spotting 'aliens'. During some trials, a speaker's face flashed green for $1 \mathrm{~s}$. If participants detected this change, they were to say 'Alien!' instead of 'true' or 'false' during the question period. In all other aspects, the experiment was the same as Experiment 3.

The intent of this study was to ensure that visual attention was being directed toward the visual event of the fact presentation while oculomotor coordinates were kept constant in the center of the screen. In this way, we attempted to test whether attention in the absence of eye movements was sufficient to produce a representation that would cause a look to the relevant region during the question period.

\subsection{Method}

\subsubsection{Participants}

Fifteen Cornell undergraduates took part in the study in exchange for course credit. All had normal vision, or vision corrected by soft contact lenses.

\subsubsection{Stimuli and procedure}

The method was exactly the same as Experiment 3, with the addition of the alien task. Participants were told that on a proportion of the trials (it was, in fact, two out of eight) one of the speakers would be an alien. This would be revealed by the speaker's face turning green for $1 \mathrm{~s}$ (this effect was achieved by altering the color balance on a section of the MPEG). If the participant saw an alien during the course of a trial, they were to answer 'alien' instead of 'true' or 'false' during the answer period. Thus, we motivated participants to attend to aliens by pointing out that if they saw one, they didn't have to bother remembering the rest of the facts. Participants were given a practice trial in which an alien was present. 


\subsection{Results}

Participants detected the aliens $77 \%$ of the time with no false alarms, suggesting that they were indeed directing substantial attention to the parafoveal events.

The data were coded in the same way as Experiment 1. An average of 1.6 fixations were made during each trial. The repeated measures ANOVA of mean fixations per trial showed that the effect of port did not approach significance $(F(3,42)=0.238$, $P>0.85$ ). This suggests that the alien manipulation did little to change the participants' looking behavior, as seen in Experiment 3. The mean fixations per trial are summarized in Fig. 7.

In the analysis of the accuracy of participants' answers, four participants never looked at the critical port during the question and answer periods, and therefore had to be excluded. When participants made zero looks to the critical port their accuracy was slightly lower $(67 \%)$ than when they made one or more looks $(73 \%)$. However, this effect did not approach significance $(F(1,10)=0.106, P>0.7)$.

\subsection{Discussion}

It appears that participants were directing visual attention to the presentation of the MPEG event sufficiently to detect a brief change in color distribution. In the absence of a fixation, however, this covert orientation of attention was insufficient to produce a saccade to the relevant region during the question period.

Thus, we might conclude that the effect seen in Experiments 1 and 2 requires a fixation in the relevant location and that the eye movement to a blank region during

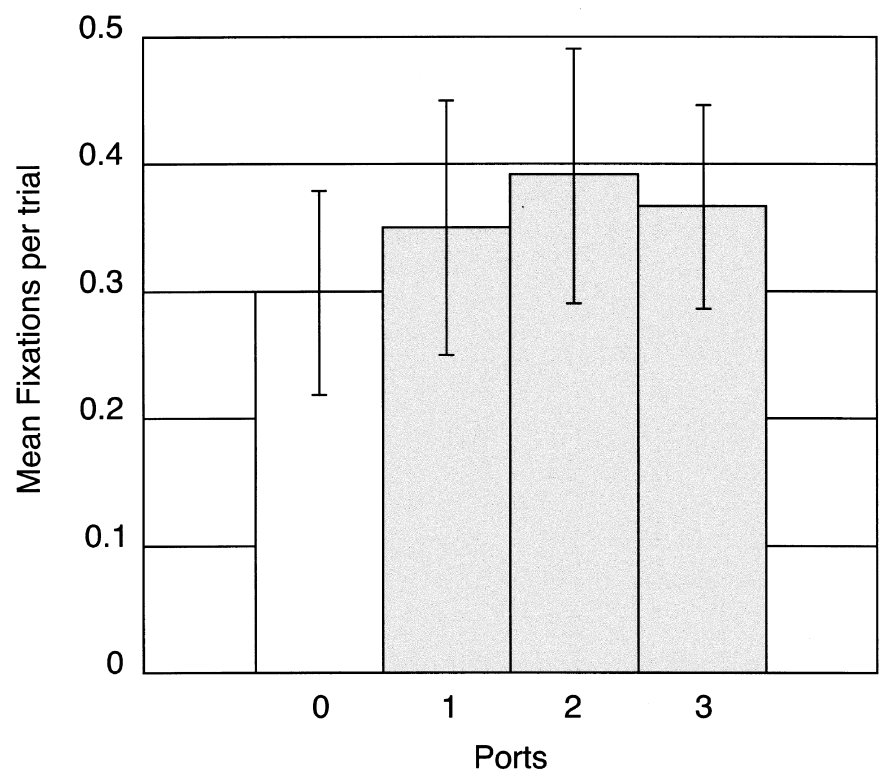

Fig. 7. Mean fixations per trial, Experiment 4 (error bars represent pooled standard errors). 
recall is mediated by a trace of the oculomotor coordinates, rather than some spatial representation that can be generated by attention alone. There are at least three objections to this conclusion.

Firstly, Experiments 3 and 4 found no evidence of an effect; that is to say, we obtained a null result. It might be bad methodological practice then to reason that the result of Experiment 1 is purely due to the presence of a fixation within the ports during presentation of the facts. Secondly, it is unclear exactly how the 'alien' manipulation is affecting participants' attention: whether the presentations are endogenously attended parafoveally for aliens, or whether participants largely ignore the MPEGs and rely on the abrupt color change to exogenously capture their attention.

Thirdly, it can be argued that fixation control during fact presentation is not the only important difference between the first two and the last two experiments. To be sure, a fixation on the presentation event brings in considerably more information than attending to the event parafoveally. So, it could be that it is not oculomotor coordinates, per se, that are stored during presentation and then triggered during the question. Rather, it could be that the body of information that the fixation gathers allows for a richer memory representation of the presentation of the fact. The question then activates this representation, and the spatial information associated with it is enough to launch an eye movement. That is, perhaps there is a kind of threshold of moderate attention necessary for this effect, and parafoveal attention is not sufficient.

Given the concerns over explicitly manipulating participants' fixations and interpreting the role of attention, we decided to abandon the method of parafoveal viewing. Since the question of import is the contribution of oculomotor information in establishing spatial tags, in the final experiment we attempted to experimentally separate spatial information and oculomotor information. If, as Winograd and Church (1988) suggest, spatial information is simply acting as a useful cue in memory tasks, then we might expect a look to the critical port when spatial (nonmotor) information is given. A purely Hebbian account (e.g. Hebb, 1968; Pulvermüller, 1999), on the other hand, might suggest that the probe question causes a reactivation of an oculomotor pattern present at encoding; thus, it would predict a look to the critical port only if it was fixated on during presentation.

\section{Experiment 5}

In this experiment, spatial information was differentiated from eye movement information using an animated 'virtual window' (see Fig. 8). ${ }^{3}$ In the 'tracking' condition, a dark blue mask with a square window in the center moved to the four ports of the $2 \times 2$ grid. This allowed participants to move their eyes to the separate locations and attend to the fact presentation. In this way, the tracking condition is essentially a replication of Experiment 2 with a blue mask over unused

\footnotetext{
${ }^{3}$ This type of display is reminiscent of the 'gun barrel' opening sequence to the James Bond films (thanks to Reuben Saunders for pointing this out).
} 
parts of the screen. In the 'watching' condition, the hole stayed in the middle of the screen, and the grid moved behind it. In this way, each of the ports came into view foveally and the participant was presented with the fact, but their oculomotor coordinates did not change. In both conditions, after the presentation, the square window expanded outwards to show the entire grid and the question was given.

Exactly the same spatial information is being presented to the fovea in both the tracking and watching conditions. Indeed, they differ only in their absolute motion: within the viewing window the animation is the same. ${ }^{4}$ Therefore, the only difference between the two conditions is the presence or absence of fixations to different absolute regions of space.

\subsection{Method}

\subsubsection{Participants}

Twenty-three high school students attending Cornell's Summer School program took part in the study in exchange for course credit. All had normal vision, or vision corrected by soft contact lenses.

\subsubsection{Stimuli and procedure}

Sixteen sets of four facts were constructed along the same criteria as the previous experiments. Each fact lasted approximately $4 \mathrm{~s}$, and was recorded with a single speaker as a sound file.

Sixteen animation sequences were generated for eight different sequences of ports in the tracking and watching conditions. Fig. 8 shows a schematic of the animation in the two conditions. Each animation began with a blank $2 \times 2$ grid that filled the entire screen. The mask window then closed into the center of the grid from the edges. The window then traveled to each corner (tracking), or the grid behind the window moved to bring each port into view in the center (watching). The animation between ports took $1 \mathrm{~s}$. At each port, a spinning cross was seen for $4 \mathrm{~s}$ while a fact was heard. The fact sound file and the spinning cross were synchronized such that they would be perceived as one event, as in Experiment 2. After four ports were seen, the window returned to the center and then expanded outwards, leaving the empty $2 \times 2$ grid. A question was then asked about one of the four facts selected randomly, and the participant answered 'yes' or 'no' into the microphone, and then pressed the space bar to initiate the next trial.

In each of the 16 trials, the order of the facts, the sets, and the order that the ports were visited were fully randomized.

\subsection{Results}

The data were coded in the same manner as the previous experiments. There was an average of 1.3 fixations during each trial, and the mean fixations per trial were analyzed using a repeated measures ANOVA. There was a significant effect of port

\footnotetext{
${ }^{4}$ This is most apparent in the example animations of the tracking and watching conditions which are available at http://susan.psych.cornell.edu/hollywood.html.
} 
TRACKING
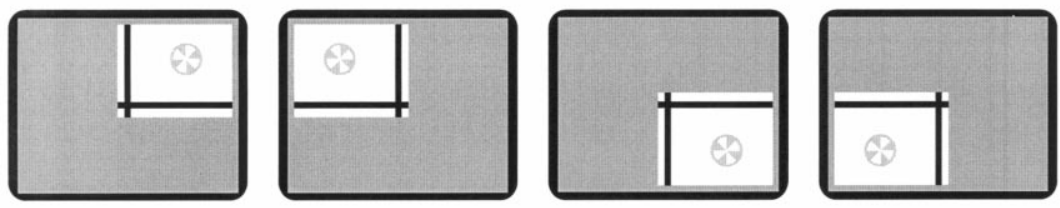

WATCHING
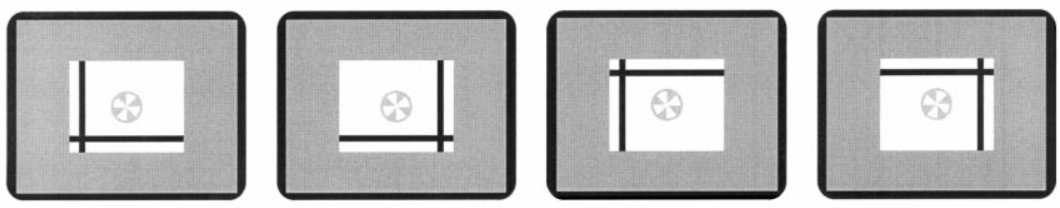

Fig. 8. Schematic of Experiment 5 tracking and watching conditions. In each condition, the viewing window is stationary for $4 \mathrm{~s}$ while a fact is heard and a spinning cross appears. Then the window animates smoothly to the next port.

$(F(3,66)=4.54, P<0.05)$, and of viewing condition $(F(1,22)=5.49, P<0.05)$. There was no evidence of an interaction $(F(3,66)=0.05, P>0.9)$. In both conditions participants had made more looks to the critical ports (mean number of looks: tracking 0.49 , watching 0.43 ) compared to the other ports (mean looks to the other three ports: tracking 0.34 , watching 0.25 ). Seventeen out of 23 participants showed this pattern of behavior. The mean number of fixations in each condition is summarized in Fig. 9. Tukey pairwise comparisons between the critical port and ports 2 and 3 reached significance (all $P<0.05$ ), although the comparison with port 1 did not reach significance $(P=0.1)$.

In the accuracy analysis, ten subjects had to be excluded because they did not make at least one look to the critical port in both conditions. The participants were slightly more accurate in the watching condition (77\%) than the tracking condition (74\%). However, this effect did not approach significance $(F(1,17)=0.40$, $P>0.5)$. There was a $1 \%$ increase in accuracy if the participants made one or more looks to the critical port, but again this effect did not approach significance $(F(1,17)=0.06, P>0.8)$.

\subsection{Discussion}

The results of the final Hollywood Squares experiment suggest that saccades to the critical port during the question period do not require fixations on separate locations in absolute space during encoding of the original event. It seems then that we might explain the null results of Experiments 3 and 4 in terms of the increased visual information that is available with a fixation, as compared to parafoveal viewing.

In retrospect, one might expect participants in both the tracking and watching 


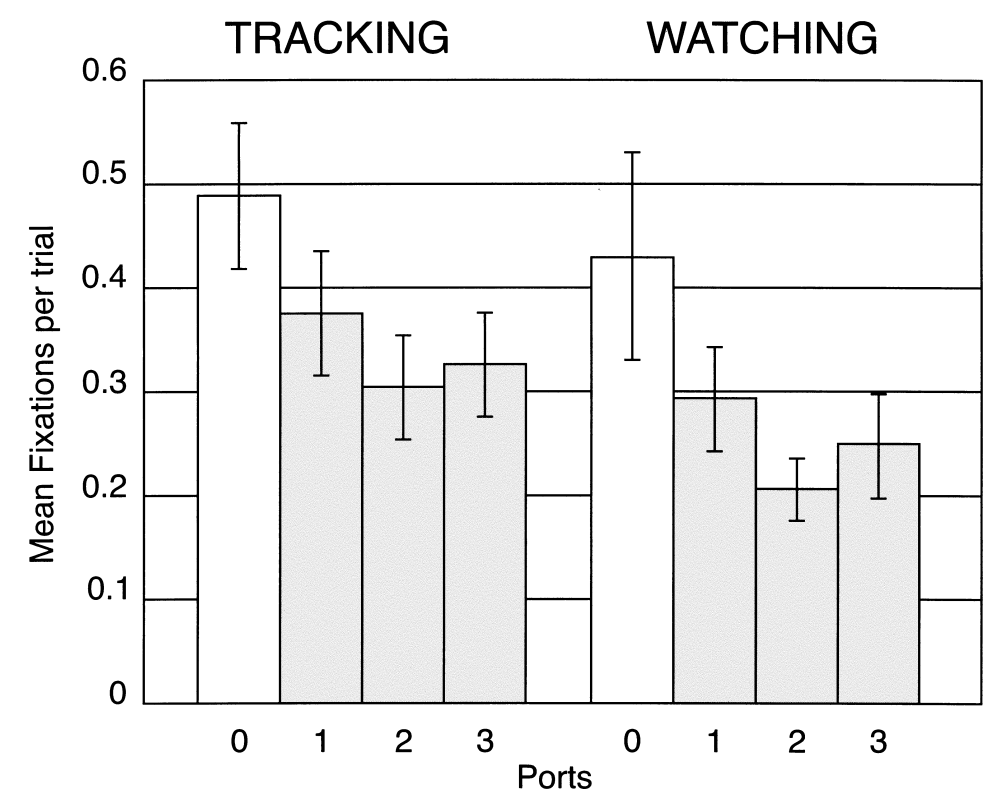

Fig. 9. Mean fixations per trial by condition, Experiment 5 (error bars represent pooled standard errors).

conditions to make saccades to the critical port, given the findings of Pylyshyn and his colleagues. Scholl and Pylyshyn (1999) argue that participants can track multiple moving items through occlusions by use of FINST visual indexes, which can maintain the identity or objecthood of up to four or five items (see also Lachter \& Hayhoe, 1995). In the watching condition, it is conceivable that participants could represent the relative locations of the four ports as they moved behind the mask, and that semantic information could be attached to these FINST indexes. In the tracking condition, the FINST indexes might also be supported by oculomotor information, since the participant makes distinct fixations at each location. This additional information could explain why there was a significant main effect of viewing condition, and a higher number of looks overall in the tracking condition.

\section{General discussion}

The experiments presented here demonstrate a new paradigm for examining the role of spatial information and partial representation in memory as revealed by eye movements. The findings raise three different questions, which form the basis of the following discussion. First we can ask, How is visual spatial information tied to the event of a heard semantic fact? There is a large body of literature investigating the role of spatial location in visual perception and memory. We will use this to try and explain the association between location and the fact presentation, and the independence of location memory and accuracy of the factual recall. Secondly, given that 
Experiment 5 suggests that location is not coded solely in the form of oculomotor coordinates, we can draw on other behavioral and neurophysiological data and ask, In what form is the spatial information represented? Lastly, the naive question, Why would people look at blank regions of space? leads to some interesting speculations and connections to the notion of the world as an 'external memory store' (O'Regan, 1992).

\subsection{How is visual spatial information tied to the event of a heard fact?}

There is strong evidence that spatial information can be learned implicitly (Chun \& Jiang, 1998; Howard, Mutter \& Howard, 1992; Mayr, 1996). Researchers such as Van der Hejiden support 'position-special' theories of visual processing (Van der Heijden, 1993), arguing that an analysis of conditional probabilities in some tasks reveals that there is no object identity information without position information (Brouwer \& Van der Heijden, 1997). Similarly, Winograd and Church (1988) found that when participants were told to remember face-name pairs presented in various locations, memory for location was superior to their recall of names, even though this information was explicitly irrelevant in the study phase. Moreover, Kennedy and Murray (1987) found that when reading, participants could make accurate, long-range regressive saccades to queried words. It seems uncontroversial then that spatial location is often encoded during recall and recognition tasks. However, it is debated whether this spatial information is encoded automatically in memory tasks (Hasher \& Zacks, 1979), or whether it is a function of the task and the types of items being remembered.

Andrade and Meudell (1993) report that participants' memory for the location of words presented in the four corners of a screen is unaffected by the difficulty of a concurrent counting task, or the degree to which participants are instructed to focus on either task. However, Naveh-Benjamin (1988) claims that whereas the spatial representations of objects are more or less automatic and not susceptible to task manipulations, word location memory can be influenced by many factors supposedly not relevant to automatic processes (by the criteria of Hasher \& Zacks, 1979). Zimmer (1998) found that recognition judgments for items was impaired by incongruency of spatial layout in study and test periods. This effect was found when words and picture trials were mixed, but not for words when in 'modality pure' blocks. Similarly, Pezdek et al. (1986) found that item memory was affected independently from location memory for words but not for pictures.

In general then, previous research suggests two dissociations that may be useful in accounting for the Hollywood Squares data. First, object location is encoded with a high degree of automaticity, whereas memory for word location is susceptible to task demands and, perhaps, is encoded by a separate process. Second, there is some evidence that memory for item information seems to be coded fairly independently of location information. Pollatsek et al. (1990) found that a preview aided object naming regardless of whether the target was in the same location or more than $5^{\circ}$ apart. There is also evidence for separate buffers for spatial and object information 
from PET studies (Smith, Jonides, Koeppe, Awh, Schumacher \& Minoshima, 1995) and ERP studies (Ruchkin, Johnson, Grafman, Canoune \& Ritter, 1997).

These dissociations suggest the following account of our data. The probe question actives a memory trace for the event of the factual presentation. Associated with the visual components of that memory trace (an MPEG video or a spinning cross) is a representation of its spatial location, and sufficient activation of that representation launches an eye movement. However, the research discussed above suggests that this spatial trace would do little to strengthen or improve memory for the semantic information. Hence, accuracy in our experiments does not improve with looks to the critical port. In other words, the implicit aspect of memory examined by these experiments is independent of the explicit memory of participants for the semantic facts. ${ }^{5}$

We would argue then that the phenomena revealed in our experiments (and, for example, those of Cohen et al., 1999) cannot be accounted for purely in terms of contextual memory effects whereby all relevant information associated with a memory for the fact - including spatial location - is reactivated upon recollection, since accuracy in one (looks to the critical port) is not correlated with accuracy in the other (answers to the factual question).

One could ask, however, why should the saccadic patterns we observe be due to an implicit aspect of memory, given that in general eye movements can be either automatic processes or, to some degree, voluntary actions. After all, in Experiments 3 and 4, we asked subjects to deliberately maintain fixation on a spinning cross, despite events occurring parafoveally. However, it is widely accepted in visual psychophysics that this endogenous control over eye position is tentative at best. For example, experiments in which participants are instructed to maintain eye fixation during some task often require eye tracking in order to be accepted in the literature. Moreover, in proposing a model of saccade generation, Findlay and Walker (1999, p. 664) note that although an individual can override lower level processes and move their eyes voluntarily, "such actions are considered unusual", and that when "more than one target could be selected for gaze redirection... conflict resolution is only resolved in this implicit way with no overriding supervisory decisions". Below we discuss other work which uses eye movements as a window on implicit cognitive processing. Overall, the consistent pattern of behavior across participants, the typical lack of conscious control of eye movements and explicit memory for location, argues against the explanation of participants intentionally, or strategically, looking at the blank port where they know the fact occurred.

There is an interesting connection between the implicit aspect of memory investigated here and previous research on spatial encoding and explicit memory. For example, in Experiment 2 in Andrade and Meudell (1993), participants were

\footnotetext{
${ }^{5}$ Future work will address the question whether semantic memory can be improved if each fact can be associated with a unique location. That is to say, over the course of each experiment, the same four locations were used in every trial, resulting in some eight to 16 facts being associated with each corner of the screen. It is possible that, in the long term, the spatial information conveyed by a look to a critical port was not distinct enough to improve a memory trace for the relevant fact in that particular trial.
} 
presented with groups of four words appearing sequentially in random corners of a screen. Later, they were asked to identify and then place words in their correct location. In a 'final comment' of the paper, the researchers note that participants gave the correct location reliably above chance about a third of the time, but often reported that they were guessing throughout the whole spatial component of the memory task. In our studies, where location is never explicitly relevant to the task, about a third of all the participants' looks were to the critical port, the 'correct location'. We might tentatively suggest that if Andrade and Meudell's participants had simply reported the location that they found themselves looking at most often, this would fit with both their level of accuracy and lack of explicit knowledge for location.

In summary then, the Hollywood Squares experiments have provided further evidence that supports the dissociations between spatial encoding and explicit memory of items. Even though spatial location is irrelevant in the task and measured with eye movements (behavioral responses of which the participant has little awareness or conscious control), spatial information is nonetheless encoded during perception of the visual event. Moreover, this information can be reactivated by accessing a semantic, auditory feature of that event. It has further been shown that the location information attaches to the event as a visual object, and hence accuracy on recall of that semantic information is not improved with access to spatial properties. $^{6}$

\subsection{In what form is the spatial information represented?}

There is a large body of behavioral and neurophysiological evidence that converges on a view of the primate visual system as comprised of two subsystems or information streams (Mishkin, Ungerleider \& Macko, 1983). The current picture, as conveyed by Goodale and Milner (1992) and others, is of a 'how' and a 'what' system. The 'how' pathway is based in the dorsal visual pathway and is a pragmatic, action-based system controlling sensorimotor output in an egocentric reference frame (Milner \& Goodale 1995). In contrast, the 'what' system in the ventral pathway utilizes an allocentric framework (Dijkerman, Milner \& Carey, 1998) and is concerned with recognition, categorization and other cognitive functions.

Eye movements are planned and executed in the dorsal pathway (Colby \& Goldberg, 1999; Corbetta et al., 1998; Rossetti, 1998). However, there are several reasons why the effect observed in the experiments presented here must be at least partially mediated by input from the ventral pathway. First, the effect cannot be explained in terms of egocentric oculomotor coordinates, given that representations of oculomotor movements appear to be short-lived and are not stored (Goodale \& Humphrey, 1998). Second, the results of Experiment 5 suggest that fixations to distinct locations

\footnotetext{
${ }^{6}$ Note that this further conclusion might be troubled if looks to the critical location were found when the facts were purely auditory events that were localized by stereo position. Also, though we found that looks to the 'critical port' did not appear to increase accuracy, it could be the case that if participants' fixations were experimentally controlled during the question period - i.e. they were instructed to look at a noncritical port - their performance might be impaired.
} 
in space are not required to cause systematic saccades to these blank regions. The perceptual system is capable of using the purely spatial information foveated in one location (watching condition of Experiment 5) to build up a representation of the grid that will cause saccades to critical ports during recall. Lastly, Spivey and Geng (2000) report interesting data on the importance of a background grid in generating the saccades to blank regions of space. If the participants were presented with a completely blank background screen during the question period, there were considerably fewer looks to the critical port compared to a grid such as the one used in the Hollywood Squares experiments. ${ }^{7}$ This suggests that, in some sense, participants are exploiting an external structure in their internal spatial representation, which would again implicate the allocentric ventral pathway. ${ }^{8}$

Much of the behavioral and neurophysiological research in this area has been directed toward finding dissociations between the two visual systems. However, in the case of the experiments presented here, there is a clear case of significant merging of the two information streams, such as found by Deubel, Schneider and Paprotta (1998), who report evidence of "obligatory and selective coupling of dorsal processing for saccade programming and ventral processing for perception and discrimination" (see also Bridgeman \& Huemer, 1998; Bridgeman, Peery \& Anand, 1997; Dijkerman et al., 1998; Rossetti, 1998).

\subsection{Why would people look at blank regions of space?}

Before answering this question, we shall briefly look at an emerging field of eye movement research, and then apply some of the reasoning employed there to the current set of experiments.

There is a growing body of literature that uses online measures of eye movements in complex natural tasks to reveal subtle, partial activation of representations during the time course of cognitive processing. A particularly fruitful area has been language comprehension. Tanenhaus et al. (1995) have demonstrated via eye movements that even in the early moments of language processing, listeners can use visual context cues to disambiguate syntactic structures. Spivey and Marian (1999) show that in

\footnotetext{
${ }^{7}$ In Section 1, we referred to a paper by Glenberg et al. (1998) which found that participants averted their gaze during a memory task. They demonstrated that the behavior was not due to social factors, but rather it was indicative of the cognitive system disengaging an 'environment monitoring system' to devote more resources to the memory task. As noted, this seems superficially at odds with our findings. However, the discussion of external reference frames leads us to speculate that perhaps the difference between participants looking to and looking away from their surroundings is determined by the relevance of externally available structures and information. In Glenberg et al.'s study, participants were asked math questions, or what they did a week previously. In contrast, our questions were related to information the participants had been given in their current context. This distinction suggests that an interesting line of inquiry would be to investigate under what conditions could external structures facilitate internal cognitive processes, and what conditions would force the cognitive system to disengage from the environment.

${ }^{8}$ In future work, we will have participants watch the four fact events on one computer screen, and then look at another screen with an identical $2 \times 2$ grid during the question period. If we still found the effect of saccades to the (isomorphic) critical port, that would suggest that participants normally transfer their relative spatial representations onto different absolute locations in the world.
} 
bilinguals, instructions to pick up an object can partially activate a representation of another object with similar initial phonology in a different language. Experiment 2 of Keysar, Barr, Balin and Paek (1998) found that when a confederate involved in a drawing task with a participant asked about a picture, the participant sometimes made a saccade to a similar picture, even though that item was clearly outside the "common ground' of the discourse (not visible to the collaborator). Lastly, there is emerging evidence that participants passively listening to a story with a certain directionality (for example, a train moving from right to left) will make eye movements that cluster along that axis when they are looking at a blank display, or even when their eyes are closed (Spivey, Tyler, Richardson \& Young, 2000).

These studies demonstrate that within the time course of a cognitive act, a participant may look to an item that is irrelevant by certain constraints (e.g. the syntactic structure, the bilingual's language mode, common ground) because the representation of that item is partially active. This is consistent with recent neurophysiology work in which microstimulation of the frontal eye field in a motion detection task revealed that decision formation and oculomotor preparation share a common preparational level of neural organization (Gold \& Shodlen, 2000). Thus, one might expect to observe intermediate (or implicit) stages of decision formation in oculomotor signals. Furthermore, work by Ballard et al. (1995) shows that in a block moving task, participants' looking patterns reveal that they are minimizing the demands on their short-term memory, and instead casting frequent looks to the various task areas. Moreover, Hayhoe, Bensinger and Ballard (1998) found that transaccadic changes to the colors of the blocks often went unnoticed, suggesting that participants were relying on the stability of the external information instead of storing it in memory. Blackmore, Brelstaff, Nelson and Trosciano (1995), Levin and Simons (1997) and O'Regan, Rensink and Clark (1999), among others, offer further compelling evidence of this with the 'change blindness' effect, demonstrating that dramatic changes in object properties during an eye movement or temporary occlusions are often not detected by the participant.

These studies can be incorporated into a general account of the visual system as utilizing an 'external memory store' (O'Regan, 1992). The notion is that our phenomenology of perceiving a visually rich and complete world is largely an illusion: parafoveally we do not have access to fine grained detail, and we store very little visual information about our surroundings between saccades (Bridgeman et al., 1994). This phenomenological illusion is maintained by frequent saccades that bring any objects activated in cognition into foveated vision. All that is needed is some spatial tag such that we know where to look in order to get more information on objects involved in a cognitive process. Ballard et al. (1997) give a more formal account of this notion of an 'external memory store' with a theory of deictic coding. They adduce further computational arguments for the efficiency of referring to cognitive items by 'pointers' such as oculomotor coordinates. Moreover, from the background of the stimulus-response compatibility paradigm, Hommel (2000) concludes that "retrieving nonspatial information about an object leads to the facilitation of responses that spatially correspond to this object" (see also Hommel \& Knuf, 2000). 
This perspective on the interaction between vision and cognition can be applied to the experiments presented here. When participants are queried about semantic information related to a certain event, many aspects of that event will become partially activated. This will often launch a saccade to the relevant spatial location in order to gather more data. In a sense, this subsystem is 'change blind' to the fact that there is no information there and the location is empty. Hence, there are looks to the critical port, but no increase in accuracy, since fixation of the empty quadrant cannot provide any relevant semantic information. In this way, we can argue that the puzzling effect of saccades to blank regions of space is a consequence of a perceptual-motor system that relies on rapid access to an external store of information, and doesn't always know when that external store has changed.

\section{Conclusion}

Van der Heijden (1997) proposes an 'unlimited capacity' account of visual information processing in which "perception is for selection and selection is for action". In a rejoinder to Treisman's commentary (p. 360), he continues that this view is "not the complete story, but only a part of it. What is omitted is the role played by eye movements and fixations". He concludes that, since eye movements are actions, "perception is for selection, selection is for action, and action is for perception". Arguably, we have found evidence of a system that spatially indexes non-visual information, then immediately makes a saccade to the correct location when that information is pertinent but visually non-existent. In line with Van der Heijden's comments, this suggests the work of a primitive mechanism of 'action for perception'.

In our studies, spatial information is encoded in a memory for purely auditory, semantic information, even when location is never mentioned or measured explicitly in the task, and does not seem to facilitate performance. This can be interpreted in terms of the cognitive system giving a spatial tag to semantic representations, and immediately launching a saccade to the relevant location when that representation is triggered. A saccade will even be made to an entirely empty location: in a sense, part of the system is unaware of the fact that the information is no longer present. It can be argued that these systematic looks to empty regions of space are indicative of the same mechanism revealed in the looking patterns of participants in the block moving task of Ballard et al. (1997): an embodied cognitive system that employs a spatial indexing procedure and relies on the stability and availability of external information.

\section{Acknowledgements}

The authors would like to thank David Perkowski, Jeff Ng, Melinda Tyler and Holly Golden for help with data collection and coding. We are grateful to two anonymous reviewers for helpful comments, and to Ulric Neisser, Larry Barsalou and Joy Geng for valuable discussions. This research was supported by a Sloan 
Foundation Research Fellowship in Neuroscience (M.J.S.), and a Sage Fellowship from Cornell University (D.C.R.). All subjects gave informed consent.

\section{References}

Allopenna, P. D., Magnuson, J. S., \& Tanenhaus, M. K. (1998). Tracking the time course of spoken word recognition using eye movements: evidence for continuous mapping models. Journal of Memory and Language, 38 (4), 419-439.

Althoff, R. R., \& Cohen, N. J. (1999). Eye-movement-based memory effect: a reprocessing effect in face perception. Journal of Experimental Psychology: Learning, Memory, and Cognition, 25 (4), 9971010.

Andrade, J., \& Meudell, P. (1993). Short report: is spatial information encoded automatically in memory? The Quarterly Journal of Experimental Psychology, 46A (2), 365-375.

Ballard, D. H., Hayhoe, M. M., \& Pelz, J. B. (1995). Memory representations in natural tasks. Journal of Cognitive Neuroscience, 7 (1), 66-80.

Ballard, D. H., Hayhoe, M. M., Pook, P. K., \& Rao, R. P. N. (1997). Deictic codes for the embodiment of cognition. Behavioral and Brain Sciences, 20 (4), 723-767.

Bjork, R. A., \& Richardson, K. A. (1989). On the puzzling relationship between environmental context and human memory. In Izawa, \& Chizuko, Current issues in cognitive processes: the Tulane Flowerree Symposium on Cognition. Hillsdale, NJ: Lawrence Erlbaum.

Blackmore, S. J., Brelstaff, G., Nelson, K., \& Troscianko, T. (1995). Is the richness of our visual world an illusion? Transsaccadic memory for complex scenes. Perception, 24, 1075-1081.

Bradley, M. M., Cuthbert, B. N., \& Lang, P. J. (1988). Perceptually driven movements as contextual retrieval cues. Bulletin of the Psychonomic Society, 26 (6), 541-553.

Bridgeman, B. (1992). Conscious vs unconscious processes. Theory \& Psychology, 2 (1), 73-88.

Bridgeman, B., \& Huemer, V. (1998). A spatially orientated decision does not induce consciousness in a motor task. Consciousness and Cognition, 7, 454-464.

Bridgeman, B. C., Van der Heijden, A. H. C., \& Velichkovsky, B. M. (1994). A theory of visual stability across saccadic eye movements. Behavioral and Brain Sciences, 17, 247-292.

Bridgeman, B., Peery, S., \& Anand, S. (1997). Interaction of cognitive and sensorimotor maps of visual space. Perception and Psychophysics, 59 (3), 456-469.

Brouwer, R. T. F., \& Van der Heijden, A. H. C. (1997). Identity and position: dependence originates from independence. Acta Psychologica, 95, 215-237.

Chun, M. M., \& Jiang, Y. (1998). Contextual cueing: implicit learning and memory of visual context guides spatial attention. Cognitive Psychology, 36, 28-71.

Cohen, J. D., MacWhinney, B., Flatt, M., \& Provost, J. (1993). Psyscope: a new graphic interactive environment for designing psychology experiments. Behavioral Research Methods, Instruments, and Computers, 25 (2), 257-271.

Cohen, N. J., Ryan, J., \& Althoff, R. R. (1999). The eye movement-based relational manipulation effect: evidence for detailed representations of the world. Paper presented at the Tenth European Conference on Eye Movements, Utrecht University, Utrecht, The Netherlands.

Colby, C. L., \& Goldberg, M. E. (1999). Space and attention in parietal cortex. Annual Review of Neuroscience, 22, 319-349.

Corbetta, M., Akbudak, E., Conturo, T. E., Snyder, A. Z., Ollinger, J. M., Drury, H. A., Linenweber, M. R., Petersen, S. E., Raichle, M. E., Van Essen, D. C., \& Shulman, G. L. (1998). A common network of functional areas for attention and eye movements. Neuron, 21, 761-773.

Deubel, H., Schneider, W. X., \& Paprotta, I. (1998). Selective dorsal and ventral processing: evidence for a common attentional mechanism in reaching and perception. Visual Cognition, 5 (1/2), 81-107.

Dijkerman, H. C., Milner, A. D., \& Carey, D. P. (1998). Grasping spatial relationships: failure to demonstrate allocentric visual coding in a patient with visual form agnosia. Consciousness and Cognition, 7, 424-437. 
Fendrich, D. W. (1998). Recognition benefits from the reinstatement of a spatial representation of motoric processing. Psychological Research, 61, 125-134.

Findlay, J. M., \& Walker, R. (1999). A framework for saccadic eye movement control based on parallel processing and competitive inhibition. Behavioral and Brain Sciences, 22 (4), 661-721.

Glenberg, A. M., Schroeder, J. L., \& Robertson, D. A. (1998). Averting the gaze disengages the environment and facilitates remembering. Memory and Cognition, 26 (4), 651-658.

Gnadt, J. W., Bracewell, R. M., \& Andersen, R. A. (1991). Sensorimotor transformation during eye movements to remembered visual targets. Vision Research, 31 (4), 693-715.

Godden, D. R., \& Baddeley, A. D. (1975). Context-dependent memory in two natural environments: on land and underwater. British Journal of Psychology, 66 (3), 325-331.

Goodale, M. A., \& Humphrey, G. K. (1998). The objects of action and perception. Cognition, 67 (1-2), 181-182.

Goodale, M. A., \& Milner, A. D. (1992). Separate visual pathways for perception and action. Trends in Neuroscience, 15 (1), 20-25.

Hasher, L., \& Zacks, R. T. (1979). Automatic and effortful processes in memory. Journal of Experimental Psychology: General, 108, 356-388.

Hayhoe, M. M., Bensinger, D. G., \& Ballard, D. H. (1998). Task constraints in visual working memory. Vision Research, 38 (1), 125-137.

Hebb, D. O. (1968). Concerning imagery. Psychological Review, 75, 466-477.

Hommel, B. (2000). Automatic integration of spatial information. Manuscript submitted for publication.

Hommel, B., \& Knuf, L. (2000). Action related determinants of spatial coding in perception and memory. In C. Freksa, C. Habel, \& K. F. Wender (Eds.), Spatial cognition (Vol. II). Berlin: Springer (in press).

Howard Jr., J. H., Mutter, S. H., \& Howard, D. V. (1992). Serial pattern learning by event observation. Journal of Experimental Psychology: Learning, Memory, and Cognition, 18 (5), 1029-1039.

Kennedy, A., \& Murray, W. S. (1987). Spatial coordinates and reading: comments on Monk. The Quarterly Journal of Experimental Psychology, 39A, 649-656.

Keysar, B., Barr, D. J., Balin, J. A., \& Paek, T. S. (1998). Definite reference and mutual knowledge: process models of common ground in comprehension. Journal of Memory and Language, 39 (1), 120.

Lachter, J., \& Hayhoe, M. (1995). Capacity limitations in memory for visual locations. Perception, 24 (12), 1427-1441.

Levin, D. T., \& Simons, D. J. (1997). Failure to detect changes to attended objects in motion pictures. Psychonomic Bulletin \& Review, 4 (4), 501-506.

Logan, G. D. (1994). Spatial attention and the apprehension of spatial relations. Journal of Experimental Psychology: Human Perception and Performance, 20 (5), 1015-1063.

Mayr, U. (1996). Spatial attention and implicit sequence learning: evidence for independent learning of spatial and non spatial sequences. Journal of Experimental Psychology: Learning, Memory, and Cognition, 22 (2), 350-364.

Milner, A. D., \& Goodale, M. A. (1995). The visual brain in action. Oxford: Oxford University Press.

Mishkin, M., Ungerleider, L. G., \& Macko, K. A. (1983). Object vision and spatial vision: two cortical pathways. Trends in Neuroscience, 6, 414-417.

Naveh-Benjamin, M. (1988). Recognition memory of spatial location information: another failure to support automaticity. Memory and Cognition, 16 (5), 437-445.

O'Regan, J. K. (1992). Solving the 'real' mysteries of visual perception: the world as an outside memory. Canadian Journal of Psychology, 46, 461-488.

O'Regan, J. K., Rensink, R. A., \& Clark, J. J. (1999). Change-blindness as a result of "mudsplashes". Nature, 398 (6722), 34.

Pezdek, K., Roman, Z., \& Sobolik, K. G. (1986). Spatial memory for objects and words. Journal of Experimental Psychology: Learning, Memory, and Cognition, 12 (4), 530-537.

Pollatsek, A., Rayner, K., \& Henderson, J. M. (1990). Role of spatial location in integration of pictorial information. Journal of Experimental Psychology: Human Perception and Performance, 16 (1), 199210.

Pulvermüller, F. (1999). Words in the brain's language. Behavioral and Brain Sciences, 22, 253-279. 
Pylyshyn, Z. W. (1989). The role of location indexes in spatial perception: a sketch of the FINST spatial index model. Cognition, 32, 65-97.

Pylyshyn, Z. W. (1994). Some primitive mechanisms of spatial attention. Cognition, 50, 363-384.

Pylyshyn, Z. W., \& Storm, R. W. (1988). Tracking multiple independent targets: evidence for a parallel tracking mechanism. Spatial Vision, 3 (3), 1-19.

Rossetti, Y. (1998). Implicit short-lived motor representations of space in brain damaged and healthy patients. Consciousness and Cognition, 7, 520-558.

Ruchkin, D. S., Johnson Jr., R., Grafman, J., Canoune, H., \& Ritter, W. (1997). Multiple visuospatial working memory buffers: evidence from spatiotemporal patterns of brain activity. Neuropsychologia, 35 (2), 195-209.

Scholl, B. J., \& Pylyshyn, Z. W. (1999). Tracking multiple items through occlusion: clues to visual objecthood. Cognitive Psychology, 38, 259-290.

Sinclair, G. P., Healy, A. F., \& Bourne Jr., L. E. (1997). The acquisition and long term retention of temporal, spatial and item information. Journal of Memory and Language, 36, 530-549.

Smith, E. E., Jonides, J. M., Koeppe, R. A., Awh, E., Schumacher, E. H., \& Minoshima, S. (1995). Spatial versus object working memory: PET investigations. Journal of Cognitive Neuroscience, 7 (3), 337356.

Spivey, M. J., \& Geng, J. (2000). Perceptual-motor mechanisms in visual memory: saccades to objects in memory. Manuscript submitted for publication.

Spivey, M. J., \& Marian, V. (1999). Cross talk between native and second languages: partial activation of an irrelevant lexicon. Psychological Science, 10 (3), 281-284.

Spivey, M. J., Tyler, M. J., Richardson, D. C., \& Young, E. (2000). Eye movements during comprehension of spoken scene descriptions. Proceedings of the 22nd Annual Conference of the Cognitive Science Society. Mahwah; NJ: Erlbaum.

Tanenhaus, M. K., Spivey-Knowlton, M., Eberhard, K., \& Sedivy, J. (1995). Integration of visual and linguistic information during spoken language comprehension. Science, 268, 1632-1634.

Van der Heijden, A. H. C. (1993). The role of position in object selection in vision. Psychological Research, 65, 44-58.

Van der Heijden, A. H. C. (1997). Two stages in visual information processing and visual perception? Visual Cognition, 3 (4), 325-361.

Winograd, E., \& Church, V. (1988). Role of spatial location in learning face-name associations. Memory and Cognition, 16 (1).

Zimmer, H. D. (1998). Spatial information with pictures and words in visual short-term memory. Psychological Research, 61, 277-284. 